\title{
ON LIN'S CONDITION FOR PRODUCTS OF RANDOM VARIABLES WITH SINGULAR JOINT DISTRIBUTION
}

\author{
BY
}

\author{
ALEXANDER IL'INSKII (KHARKOV) AND SOFIYA OSTROVSKA (ANKARA)
}

\begin{abstract}
Lin's condition is used to establish the moment determinacy/indeterminacy of absolutely continuous probability distributions. Recently, a number of papers related to Lin's condition for functions of random variables have appeared. In the present paper, this condition is studied for products of random variables with given densities in the case when their joint distribution is singular. It is proved, assuming that the densities of both random variables satisfy Lin's condition, that the density of their product may or may not satisfy this condition.
\end{abstract}

2020 Mathematics Subject Classification: Primary 60E05; Secondary $44 \mathrm{~A} 60$.

Key words and phrases: random variable, singular distribution, Lin's condition.

\section{INTRODUCTION}

Lin's condition plays a significant role in the investigation of the moment determinacy of absolutely continuous probability distributions. Generally speaking, it is used along with Krein's logarithmic integral to establish the so-called 'converse criteria'. See, for example, [4, Section 5, Theorems 5-10] and [7, Section 2]. This condition was introduced and applied by G. D. Lin [3], while the name 'Lin's condition' was put forth in [10]. Let us recall the pertinent notions.

DEFINITION 1.1. Let $f$ be a probability density continuously differentiable on $(0,+\infty)$. The function

$$
L_{f}(x):=-\frac{x f^{\prime}(x)}{f(x)}
$$

is called Lin's function of $f$.

Formula (1.1) implies that Lin's function of $f$ is defined only at the points where $f$ does not vanish. In this article, we consider the probability densities of positive random variables whose Lin's functions are defined for all $x>0$. 
DEFINITION 1.2. Let $f \in C^{1}(0,+\infty)$ be a probability density of a positive random variable. We say that $f$ satisfies Lin's condition on $\left(x_{0},+\infty\right)$ if $L_{f}(x)$ is increasing on $\left(x_{0},+\infty\right)$ and $\lim _{x \rightarrow+\infty} L_{f}(x)=+\infty$.

In [7], it was shown that if a density $f$ satisfies Lin's condition, then $f$ belongs to the Karamata class of rapidly varying functions of index $-\infty$. In addition, some generalizations of Lin's results for such densities were obtained in connection with the 'converse' conditions for the moment determinacy of probability distributions. Due to the importance of this condition for establishing the moment (in)determinacy of absolutely continuous probability distributions (see [4], [8] and references therein), it is of interest how various operations on random variables affect Lin's condition. Recently, Kopanov and Stoyanov in [2] proved that if a density $f$ of a positive random variable $X$ fulfills Lin's condition, then the densities of $X^{r}, r>0$, and of $\ln X$ have this property, too. In addition, if $L_{f}(x) / x \rightarrow+\infty$ as $x \rightarrow+\infty$, then the density of $e^{X}$ also satisfies Lin's condition.

The moment problem for products of random variables naturally leads to considering Lin's condition for the densities of products. In [2], it was asked whether Lin's condition is inherited by the products of random variables whose densities satisfy Lin's condition. It was conjectured that the answer is affirmative in the case of an absolutely continuous joint distribution. While this statement holds for independent random variables, the results of [1] show that it may not be true in general. For more information on the moment problem for products we refer to [4, Section 6] and [5], [6], [9].

In the present paper, this problem is investigated for the case when the joint distribution of factors $\xi_{1}$ and $\xi_{2}$ is singular rather than absolutely continuous. More precisely, the following question is examined: assuming that the densities of both $\xi_{1}$ and $\xi_{2}$ satisfy Lin's condition, their joint distribution is singular, and Lin's function of the product $\xi_{1} \cdot \xi_{2}$ exists, can it be claimed that the density of $\xi_{1} \cdot \xi_{2}$ satisfies Lin's condition? Although it is so for some natural examples, this work reveals that even for the simplest case when $f_{1}=f_{2}=f$ satisfies Lin's condition, Lin's function of the product may exhibit a rather 'hectic' behaviour. This is demonstrated in the next statement, which is the main result of this paper.

THEOREM 1.1. Let $f_{1}$ and $f_{2}$ be densities of positive random variables, both having Lin's functions on $(0,+\infty)$. Then there exists a random vector $\left(\xi_{1}, \xi_{2}\right)$ with a singular distribution $P$ and satisfying the conditions below:

(1) $\xi_{1}$ and $\xi_{2}$ have densities $f_{1}$ and $f_{2}$, respectively;

(2) the density $g$ of the product $\xi_{1} \cdot \xi_{2}$ is continuously differentiable on $(0,+\infty)$;

(3) the following equalities hold:

$$
\limsup _{x \rightarrow+\infty} L_{g}(x)=+\infty, \quad \liminf _{x \rightarrow+\infty} L_{g}(x)=-\infty .
$$


REMARK 1.1. Clearly, equalities (1.2) imply that $g$ does not fulfill Lin's condition on any interval $\left(x_{0},+\infty\right)$, so assuming that the densities of $\xi_{1}$ and $\xi_{2}$ satisfy Lin's condition on $(0,+\infty)$, we deduce that, in general, this property is not inherited by their product $\xi_{1} \cdot \xi_{2}$ when the joint distribution of $\xi_{1}$ and $\xi_{2}$ is singular.

\section{PROOF OF THE MAIN THEOREM}

We start with the construction of a singular distribution with given marginal positive densities, which will be used in what follows.

LEMMA 2.1. Let $f_{1}$ and $f_{2}$ be continuous probability densities positive on $(0,+\infty)$ and vanishing elsewhere. Then there exists a singular distribution $\tilde{P}$ concentrated on a curve $x_{2}=\varphi\left(x_{1}\right)$ such that the projections of $\tilde{P}$ on the coordinate axes have the given densities $f_{1}\left(x_{1}\right)$ and $f_{2}\left(x_{2}\right)$. Here, $\varphi$ is a continuously differentiable strictly increasing function.

Proof. Let $F_{1}$ and $F_{2}$ be the distribution functions with densities $f_{1}$ and $f_{2}$, respectively. By the assumptions of the lemma, both $F_{1}$ and $F_{2}$ are continuously differentiable and strictly increasing, hence so are their quantile functions. Using the quantile $Q_{2}=F_{2}^{-1}$, define

$$
\varphi(x):=Q_{2}\left(F_{1}(x)\right), \quad x>0 .
$$

Now, for a random variable $\xi_{1}$ with distribution function $F_{1}$, set $\xi_{2}=\varphi\left(\xi_{1}\right)$. Then $\xi_{2}$ has density $f_{2}$ because

$$
F_{\xi_{2}}(x)=\mathbf{P}\left\{\varphi\left(\xi_{1}\right) \leqslant x\right\}=\mathbf{P}\left\{F_{1}\left(\xi_{1}\right) \leqslant F_{2}(x)\right\}=F_{2}(x), \quad x>0,
$$

due to the fact that $F_{1}\left(\xi_{1}\right)$ is uniformly distributed on $(0,1)$.

Next, consider the random vector $\left(\xi_{1}, \xi_{2}\right)$. Obviously, it has a singular distribution, say $\tilde{P}$, concentrated on the curve

$$
K:=\left\{\left(x_{1}, x_{2}\right): x_{2}=\varphi\left(x_{1}\right)\right\}
$$

while its coordinates have the prescribed densities. It can be derived from (2.1) that

$$
\varphi^{\prime}\left(x_{1}\right)=\frac{f_{1}\left(x_{1}\right)}{f_{2}\left(\varphi\left(x_{1}\right)\right)}
$$

for all $x_{1}$. Therefore, $x_{2}=\varphi\left(x_{1}\right)$ is a solution to the initial value problem

$$
\frac{d x_{2}}{d x_{1}}=\frac{f_{1}\left(x_{1}\right)}{f_{2}\left(x_{2}\right)}, \quad x_{2}(0)=0 .
$$

Since $\varphi^{\prime}\left(x_{1}\right)>0$ for all $x_{1} \in(0,+\infty)$, it follows that $\varphi$ is strictly increasing. 
EXAMPLE 2.1. If $f_{1}=f_{2}$, that is, $\xi_{1}$ and $\xi_{2}$ are identically distributed, then $K$ is the straight line $x_{2}=x_{1}$.

LEMMA 2.2. Let $\left(\xi_{1}, \xi_{2}\right)$ be a random vector with distribution $\tilde{P}$ constructed above. Then the density $g(z, \tilde{P})$ of the product $\xi_{1} \cdot \xi_{2}$ is given by

$$
g(z, \tilde{P})=f_{1}\left(x_{1}(z)\right) \frac{x_{1}(z)}{z+\varphi^{\prime}\left(x_{1}(z)\right) x_{1}^{2}(z)},
$$

where $x_{1}(z)$ is a unique solution of the equation $x_{1}(z) \varphi\left(x_{1}(z)\right)=z$.

Proof. For every $z>0$, we denote by $\Gamma_{z}$ the set of hyperbolae in the first quadrant

$$
\Gamma_{z}:=\left\{\left(x_{1}, x_{2}\right): x_{1}>0, x_{2}>0, x_{1} x_{2}=z\right\} .
$$

Given $z>0$, denote $K \cap \Gamma_{z}=\left\{\left(x_{1}(z), x_{2}(z)\right)\right\}$. Obviously, $x_{1}(z)$ and $x_{2}(z)$ are continuous strictly increasing functions on $(0,+\infty)$. The curve $K$ has the following parametric representation in terms of $z$ :

$$
\overrightarrow{\mathbf{x}}(z)=\left(x_{1}(z), x_{2}(z)\right)=\left(x_{1}(z), \varphi\left(x_{1}(z)\right)\right) .
$$

To find the distribution function $G(z ; \tilde{P})$ of the product $\xi_{1} \cdot \xi_{2}$, write

$$
G(z ; \tilde{P})=\mathbf{P}\left(\xi_{1} \cdot \xi_{2} \leqslant z\right)=\mathbf{P}\left(\xi_{1} \leqslant x_{1}(z)\right)=F_{\xi_{1}}\left(x_{1}(z)\right),
$$

whence

$$
g(z ; \tilde{P})=f_{1}\left(x_{1}(z)\right) \cdot x_{1}^{\prime}(z) .
$$

It has to be pointed out that $x_{1}(z)$ is a continuously differentiable function of $z$, which can be rigorously proved with the help of the Mean Value Theorem. As the proof is rather straightforward, it is omitted. Meanwhile, the differentiation of $x_{1}(z) \cdot \varphi\left(x_{1}(z)\right)=z$ leads to

$$
x_{1}^{\prime}(z)=\frac{\varphi\left(x_{1}(z)\right)}{z \varphi^{\prime}\left(x_{1}(z)\right)+\varphi^{2}\left(x_{1}(z)\right)} .
$$

Notice that the denominator on the right-hand side is strictly positive. Furthermore, with the help of (2.3) it can be shown that $\varphi^{\prime \prime}$ exists as well. Substituting the last expression for $x_{1}^{\prime}(z)$ into [2.5], one derives [2.4).

EXAMPLE 2.2. If $f_{1}=f_{2}=f$, then $K=\left\{\left(x_{1}, x_{2}\right): x_{1}=x_{2}\right\}, x_{1}(z)=$ $\sqrt{z}$, and $(2.4)$ implies that

$$
g(z, \tilde{P})=\frac{f(\sqrt{z})}{2 \sqrt{z}}
$$

whence

$$
L_{g}(z)=\frac{1}{2}+\frac{1}{2} L_{f}(\sqrt{z}),
$$

demonstrating that $g$ fulfills Lin's condition whenever $f_{1}$ does. 
Finally, we present the proof of our main result.

Proof of Theorem 1.1. To construct a singular probability distribution $P$ in $\mathbb{R}^{2}$ satisfying the conditions of Theorem 1.1 , first consider its construction inside a single rectangle $\Pi=[a, b] \times[\varphi(a), \varphi(b)]$. Denote by $Q$ the restriction of the distribution $\tilde{P}$ constructed in Lemma 2.1 to $\Pi$. Select a small $\delta>0$. Precise conditions on $\delta$ will be specified later.

Clearly, one has $a=x_{1}(s)$ and $b=x_{1}(t)$ for some $s<t$. Choose $b^{\prime}=$ $x_{1}(t-3 \delta)<b$ close to $b$ and consider a non-negative function $\tau \in C^{\infty}(0,+\infty)$ satisfying

$$
\tau(z)= \begin{cases}0 & \text { when } z \notin[t-3 \delta, t] \\ \varepsilon \sin ^{2}(\nu z) & \text { when } z \in[t-2 \delta, t-\delta]\end{cases}
$$

The behaviour of $\tau$ outside $[t-3 \delta, t] \backslash[t-2 \delta, t-\delta]$, as long as this function is non-negative and infinitely differentiable on $(0,+\infty)$, is of no importance for this proof. Here, $\varepsilon$ and $\nu$ are positive real numbers subject to certain restrictions, which will be explained below.

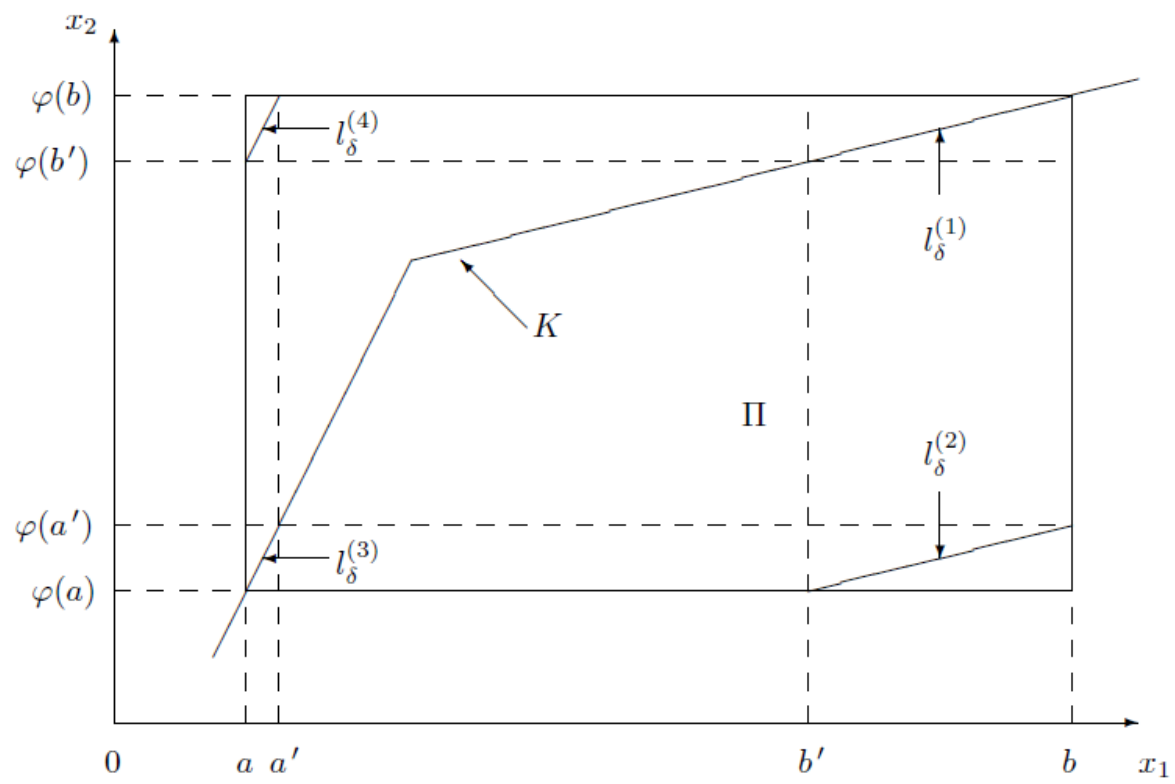

Figure 1

Recalling 2.2, consider the arc

$$
l_{\delta}^{(1)}=\left\{\left(x_{1}, x_{2}\right) \in K: b^{\prime} \leqslant x_{1} \leqslant b\right\}=\{\overrightarrow{\mathbf{x}}(z): t-3 \delta \leqslant z \leqslant t\}
$$


and consider the measure $\mu_{1}$ on this arc whose (linear) density with respect to $z$ equals $\tau(z)$. To be specific, $\mu_{1}$ is a measure concentrated on $l_{\delta}^{(1)}$ and defined in such a way that

$$
\mu_{1}\left(\left\{\overrightarrow{\mathbf{x}}(z): z_{1} \leqslant z \leqslant z_{2}\right\}\right)=\int_{z_{1}}^{z_{2}} \tau(z) d z \quad \text { whenever } \quad t-3 \delta \leqslant z_{1}<z_{2} \leqslant t .
$$

Therefore, $\mu_{1}$ is singular with respect to the Lebesgue measure in $\mathbb{R}^{2}$. In addition, its spectrum $S\left(\mu_{1}\right)$ is $l_{\delta}^{(1)}$ (recall that the spectrum $S(\lambda)$ of a measure $\lambda$ is defined by $S(\lambda):=\left\{v \in \mathbb{R}^{2}: \forall \delta>0, \lambda\left(B_{\delta}(v)\right)>0\right\}$, where $B_{\delta}(v)$ is the open disc of radius $\delta$ centered at $v \in \mathbb{R}^{2}$ ). Select $\varepsilon$ in (2.6) so that $Q-\mu_{1}$ is a (positive) measure in $\Pi$. Next, shift the mass on $l_{\delta}^{(1)}$ with density $\tau(z)$ by $d=\varphi\left(b^{\prime}\right)-\varphi(a)$ units down vertically so that the shifted $\operatorname{arc} l_{\delta}^{(2)}=l_{\delta}^{(1)}-(0, d)$ has starting point $\left(b^{\prime}, \varphi(a)\right)$ and terminal point $(b, \varphi(b)-d)$. Denote the resulting measure by $\mu_{2}$. To be more exact, for each Borel set $B \subset \mathbb{R}^{2}$, one has

$$
\mu_{2}(B)=\mu_{1}(B+(0, d))
$$

where $B+(0, d)=\left\{\left(x_{1}, x_{2}+d\right):\left(x_{1}, x_{2}\right) \in B\right\}$. The $\operatorname{arc} l_{\delta}^{(1)}$ has to be so small that $\varphi(b)-\varphi\left(b^{\prime}\right)<\varphi\left(b^{\prime}\right)-\varphi(a)$ (this is ensured by the smallness of $\delta$ ). The shifting defines a singular measure $q_{1}$ in the rectangle $\Pi$ by

$$
q_{1}(B)=\left(Q-\mu_{1}\right)(B)+\mu_{2}(B) .
$$

The construction of $q_{1}$ implies that the projections of $Q$ and $q_{1}$ on the $x_{1}$-axis are the same. However, to guarantee equal projections on both coordinate axes, the procedure has to be continued. Consider the measure $\mu_{2}$ concentrated on $l_{\delta}^{(2)}$ and find $a^{\prime}>a$ from the condition $\varphi\left(a^{\prime}\right)=\varphi(a)+\left(\varphi(b)-\varphi\left(b^{\prime}\right)\right)$. When $\delta$ is small enough, we have $a^{\prime}<b^{\prime}$. Let $\mu_{3}$ be the measure concentrated on the arc

$$
l_{\delta}^{(3)}=\left\{\left(x_{1}, x_{2}\right): x_{2}=\varphi\left(x_{1}\right), a \leqslant x_{1} \leqslant a^{\prime}\right\}
$$

and having the same projection on the $x_{2}$-axis as $\mu_{2}$. More precisely, $\mu_{3}$ is constructed in the following manner. Let $L_{c, d}:=\left\{\left(x_{1}, x_{2}\right): x_{1} \in \mathbb{R}, c<x_{2}<d\right\}$ be a horizontal strip in $\mathbb{R}^{2}$. For any $c, d$ such that $\varphi(a) \leqslant c<d \leqslant \varphi\left(a^{\prime}\right)$, where $\varphi(a)$ and $\varphi\left(a^{\prime}\right)$ are the $x_{2}$-coordinates of the endpoints of $l_{\delta}^{(3)}$, set

$$
\mu_{3}\left(L_{c, d} \cap l_{\delta}^{(3)}\right)=\mu_{2}\left(L_{c, d} \cap l_{\delta}^{(2)}\right) .
$$

Naturally, $\mu_{3}(B)=0$ whenever $B \cap l_{\delta}^{(3)}=\emptyset$. The measure $\mu_{3}$ is well-defined since the function $\varphi$ is strictly increasing. Notice that when $\varepsilon>0$ is small enough, $Q-\mu_{3}$ is a measure on $l_{\delta}^{(3)}$. Finally, shift vertically the mass defined by $\mu_{3}$ on $l_{\delta}^{(3)}$ 
as a whole by $d$ units upwards, that is, define a measure $\mu_{4}(B)=\mu_{3}(B-(0, d))$ concentrated on the $\operatorname{arc} l_{\delta}^{(4)}=l_{\delta}^{(3)}+(0, d)$. As a result, we obtain a measure $\tilde{Q}$ on $\Pi=[a, b] \times[\varphi(a), \varphi(b)]$ given by

$$
\tilde{Q}:=Q-\mu_{1}+\mu_{2}-\mu_{3}+\mu_{4} .
$$

Here, $\delta$ and $\varepsilon$ are assumed to be sufficiently small to fulfill all the conditions mentioned above and to guarantee the positivity of $\tilde{Q}$. The process of constructing $\tilde{Q}$ is illustrated in Figure 1. Evidently, $S(\tilde{Q})=(\Pi \cap K) \cup l_{\delta}^{(2)} \cup l_{\delta}^{(4)}$, whence $\tilde{Q}$ is a singular measure in $\mathbb{R}^{2}$, while the configuration of $S(\tilde{Q})$ implies that there is no functional relation between $\xi_{1}$ and $\xi_{2}$. In addition, the projections of $\tilde{Q}$ on the coordinate axes coincide with the distributions of $\xi_{1}$ and $\xi_{2}$ on $[a, b]$ and $[\varphi(a), \varphi(b)]$, respectively; that is, they have the given densities $f_{1}$ and $f_{2}$ on $[a, b]$ and $[\varphi(a), \varphi(b)]$. As before, denote by $g(z, \tilde{Q})$ the density of $\xi_{1} \cdot \xi_{2}$ in the case when the joint distribution of $\xi_{1}$ and $\xi_{2}$ coincides with $\tilde{Q}$ in $\Pi$. If $\delta>0$ is so small that the $\operatorname{arcs} l_{\delta}^{(2)}$ and $l_{\delta}^{(4)}$ do not intersect $\Gamma_{z}$, then $g(z, \tilde{Q})=g(z, \tilde{P})$, where $g(z, \tilde{P})$ is given by (2.4). It remains to show that when $\nu$ is large enough, the $z$-derivative of $g(z, \tilde{Q})$ can take arbitrarily large values, both positive and negative. To see this, first notice that for $z \in[t-2 \delta, t-\delta]$, by Lemma 2.2 .

$$
g(z, \tilde{Q})=\left(f_{1}\left(x_{1}(z)\right)-\varepsilon \sin ^{2}(\nu z)\right) \frac{x_{1}(z)}{z+\varphi^{\prime}\left(x_{1}(z)\right) x_{1}^{2}(z)} .
$$

Now, consider Lin's function for the density (2.7):

$$
L_{g}(z)=-z \frac{g^{\prime}(z, \tilde{Q})}{g(z, \tilde{Q})} .
$$

For $z \in[t-2 \delta, t-\delta]$, the ratio $z / g(z, \tilde{Q})$ is bounded, while the behaviour of $g^{\prime}(z, \tilde{Q})$ is governed by $\left(\varepsilon \sin ^{2}(\nu z)\right)^{\prime}=\nu \varepsilon \sin (2 \nu z)$, which oscillates rapidly taking positive and negative values arbitrarily large in magnitude on $[t-2 \delta, t-\delta]$ when $\nu$ is sufficiently large.

Finally, in order to find the joint distribution of $\xi_{1}$ and $\xi_{2}$, consider an infinite sequence $\left\{\Pi_{n}\right\}$ of a disjoint rectangles, $\Pi_{n}=\left[a_{n}, b_{n}\right] \times\left[\varphi\left(a_{n}\right), \varphi\left(b_{n}\right)\right]$ with $a_{n} \rightarrow+\infty$. Denote by $Q_{n}, n \in \mathbb{N}$, the restriction of the measure $P$ to $\Pi_{n}$. For each $\Pi_{n}$, construct $\tilde{Q}_{n}$ as described above, making at every step the derivatives $p^{\prime}\left(z_{n}^{*}\right)$ and $p^{\prime}\left(z_{n}^{* *}\right)$ as large in magnitude as necessary but with opposite signs. To complete the picture, set

$$
P=\tilde{P}-\sum_{n=1}^{\infty} Q_{n}+\sum_{n=1}^{\infty} \tilde{Q}_{n}
$$

It is clear that the measure $P$ satisfies all conditions stated in Theorem 1.1 
Acknowledgments. The authors would like to express their sincere gratitude to the anonymous referee, who suggested the simplifications of the proofs of Lemmas 2.1 and 2.2 and whose remarks helped to improve the presentation of this article.

\section{REFERENCES}

[1] A. Il'inskii and S. Ostrovska, On Lin's condition for products of random variables, Zh. Mat. Fiz. Anal. Geom. 15 (2019), 79-85.

[2] P. Kopanov and J. Stoyanov, Lin's condition for functions of random variables and moment determinacy of probability distributions, C. R. Acad. Bulgare Sci. 70 (2017), 611-618.

[3] G. D. Lin, On the moment problems, Statist. Probab. Lett. 35 (1997), 85-90.

[4] G. D. Lin, Recent developments on the moment problem, J. Statist. Distributions Appl. 4 (2017), $1-17$.

[5] G. D. Lin and J. Stoyanov, Moment determinacy of powers and products of nonnegative random variables. J. Theoret. Probab. 28 (2015), 1337-1353.

[6] G. D. Lin and J. Stoyanov, On the moment determinacy of products of non-identically distributed random variables. Probab. Math. Statist. 36 (2016), 21-33.

[7] A.G. Pakes, Remarks on converse Carleman and Krein criteria for the classical moment problem, J. Austral. Math. Soc. 71 (2001), 81-104.

[8] D. Stirzaker, The Cambridge Dictionary of Probability and its Applications, Cambridge Univ. Press, 2015.

[9] J. Stoyanov, Counterexamples in Probability, 3rd ed., Dover Publ., New York, 2013.

[10] J. Stoyanov, Krein condition in probabilistic moment problems, Bernoulli 6 (2000), 939-949.

Alexander Il'inskii

Department of Mathematics and Informatics

Karazin National University

Kharkov, Ukraine

E-mail: iljinskii@univer.kharkov.ua
Sofiya Ostrovska

Department of Mathematics

Atilim University

06830 Incek, Ankara, Turkey

E-mail: sofia.ostrovska@atilim.edu.tr

Received 16.2.2018;

revised version 14.12.2018 